\title{
Parâmetros físicos do solo relacionados com o avanço da frente de molhamento ${ }^{1}$
}

\author{
Paulo da S. e Souza Filho², Arno B. Heldwein², João F. Zamberlan² \& Henrique C. Corrêa ${ }^{3}$
}

\section{RESUMO}

Este estudo visou contribuir, no âmbito metodológico, nas áreas da ciência do solo e da irrigação, notadamente na microirrigação na obtenção "in situ" de parâmetros físicos e hídricos do Argissolo Vermelho Distrófico arênico (PVd). Foi projetado um sistema "multigotejador" cuja aplicação está vinculada aos princípios do "Método do Gotejador" na obtenção da condutividade hidráulica K(h) e da curva de retenção de água no solo $\theta(\mathrm{h})$. Vazões aplicadas de 2,4 e $8 \mathrm{~L} \mathrm{~h}^{-1}$ e suas respectivas áreas de molhamento e de saturação, foram medidas no momento do equilíbrio dinâmico. O valor médio de sorptividade calculado foi de $2,68 \mathrm{~cm} \mathrm{~h}^{-0,5}$, sendo obtidos os valores de $\eta=2,22, \beta=0,44$ e hw $=-2,29 \mathrm{~cm}$ de $\mathrm{H}_{2} \mathrm{O}$. Usando-se os valores dos parâmetros do solo, a curva característica de retenção de água no solo (CCRAS) e a função da condutividade hidráulica em função do conteúdo de água do solo, foram estimadas e representadas graficamente. A curva característica obtida com dados experimentais de campo não foi igual àquela obtida em laboratório, conforme metodologia padrão, mas foi possível obter uma função exponencial que permite corrigir os valores da umidade volumétrica do solo.

Palavras-chave: irrigação, gotejamento, funções de pedotransferência a campo

\section{Physical parameters of soil related to wetting front advance}

\begin{abstract}
The present study aimed to contribute, in the methodological way, to the field of Soil Science and Irrigation, more specifically microirrigation (targeted irrigation), to obtain hydro-physical parameters in situ, specially the hydrodynamic functions of a Paleudalf soil. A "multidrip" system, whose application is linked to the "Drip Method" principles, was designed to obtain hydraulic conductivity $\mathrm{K}(\mathrm{h})$ and soil-water retention curve $\theta(\mathrm{h})$. The system applied flow rates of 2, 4 and $8 \mathrm{~L} \mathrm{~h}^{-1}$ and their respective areas of wetting and saturation in the dynamic equilibrium moment were measured. The mean value of sorptivity calculated was $2.68 \mathrm{~cm} \mathrm{~h}^{-0.5}$, with values of $\eta=2.22, \beta=0.44 \mathrm{e} \mathrm{hw}=-2.29 \mathrm{~cm} \mathrm{H}_{2} \mathrm{O}$. By using these values of soil parameters, the soil-water retention characteristic curve (SWRCC) and the hydraulic conductivity function as a function of the water content in the soil were estimated and graphically represented. The retention curve obtained through field experimental data did not match to the one obtained in laboratory according to standard methodology, but it was possible to obtain an exponential function which allows the correction of the volumetric soil moisture values.
\end{abstract}

Key words: irrigation, drip method, field pedotransfer functions 


\section{INTRODUÇÃO}

O dimensionamento adequado do sistema e o manejo preciso da irrigação são dependentes de fatores ambientais relacionados principalmente com demanda diária de água da atmosfera, com características morfofisiológicas, com a arquitetura da parte aérea das plantas e com as características e propriedades do solo, principalmente com a condutividade hidráulica e a capacidade de retenção de água no solo. A capacidade de armazenamento de água no solo determina o manejo da irrigação a ser adotado.

O conceito de capacidade de campo (teor de água da camada inicialmente saturada alguns dias após a infiltração) é considerado, por muitos, um critério prático e útil para o limite superior de água que um solo pode reter (Reichardt \& Timm, 2004). Segundo Mesquita \& Moraes (2004) a condutividade hidráulica saturada (Ks) é determinada pela geometria, distribuição e continuidade dos poros. Portanto, um solo com maior volume e melhor distribuição de tamanho de poros terá uma condutividade hidráulica maior. A porosidade é função da textura e da estrutura do solo. O manejo do solo pode alterar a distribuição dos poros e, por consequência, sua densidade, influindo no movimento da água e na sorptividade $(\mathrm{S})$ na fase de umedecimento.

Os métodos e as técnicas utilizadas para quantificar a Ks e S devem ser aplicados de forma que não provoquem perturbações na estrutura do solo mas forneçam valores representativos com precisão e exatidão adequadas aos objetivos do seu uso (Borges \& Libardi, 2000). Righes \& Silva (2009) definem a porosidade de um solo como sendo o volume do solo que, em condições naturais, representa o volume da fração de água e de ar.

$\mathrm{O}$ ajuste da curva de retenção de água permite maior eficiência na estimativa da lâmina de irrigação podendo-se estabelecer as tensões críticas para reposição da água no solo (Reis et al., 2006); no entanto, um dos problemas da aplicação desse conhecimento em larga escala na irrigação reside no fato das determinações serem feitas em laboratórios especializados de física dos solos, com a utilização de equipamentos relativamente caros e, sobretudo, em processos morosos.

Andrade \& Stone (2009) concluíram que a condutividade hidráulica não saturada no ponto de inflexão da curva de retenção da água do solo $(\mathrm{Ki})$ pode ser determinada a partir do índice de sorptividade (S) em que S é a declividade da curva característica de retenção da água do solo em seu ponto de inflexão. Segundo Mesquita \& Moraes (2004) a dependência entre a condutividade hidráulica saturada e propriedades frequentemente utilizadas para fazer estimativas ou correlações, como a densidade do solo, macro e micro porosidades e porosidade, é adequada, pois sua determinação deve ser realizada com medidas diretas e se utilizando modelos. Neste sentido, Shani et al. (1987) e Oliveira (1986) propuseram uma alternativa para a elaboração das curvas características da água no solo, denominada "Método do Gotejador" e utilizaram, na resolução do fluxo saturado em equilíbrio dinâmico, um solo homogêneo assumido como condição de contorno. Os autores salientam, ainda, que o método se baseou em funções $K(h)$ e $\theta(h)$ ajustadas e, portanto, as possíveis limitações dessas funções devem ser estendidas aos resultados obtidos uma vez que não são similares àquelas obtidas por metodologia padrão de laboratório.
Seki (2007) desenvolveu um programa computacional que permite estimar os determinados valores dos parâmetros de um solo e da curva de retenção de água no solo usando o ajuste não linear. Saito et al. (2009) compararam a distribuição espacial das curvas de retenção de água no solo com e sem interpolação espacial utilizando a metodologia de Seki (2007) e verificaram que a abordagem usando a interpolação espacial foi mais exata; parte-se, então, da hipótese de que é possível determinar os principais parâmetros físico-hídricos diretamente no solo sob condições de campo, de forma ágil e com baixo custo com vista a se obter as informações necessárias para planejar e executar corretamente a irrigação.

Realizou-se este trabalho com o objetivo de determinar, em campo, as curvas características de retenção de água e da condutividade hidráulica de um solo Argissolo Vermelho Distrófico arênico a partir das variáveis, vazão de água aplicada pontualmente, avanço horizontal da frente de molhamento na superfície do solo com o tempo, área superficial saturada em equilíbrio dinâmico e a sorptividade, para implantar um sistema multigotejador a fim de agilizar a determinação do avanço da frente de molhamento com o tempo, área saturada, fluxo saturado de água no solo e a sorptividade, de forma simultânea em várias repetições.

\section{Material e Métodos}

O trabalho foi desenvolvido na área experimental do Departamento de Engenharia Rural da UFSM, em Santa Maria, situada na latitude de $29^{\circ} 41^{\prime} 24^{\prime \prime} \mathrm{S}$, longitude de $53^{\circ} 48^{\prime} 42^{\prime}$ W e altitude de $92 \mathrm{~m}$, na região fisiográfica da Depressão Central Rio Grande do Sul. O clima dessa região se enquadra no tipo fundamental "Cfa", subtropical úmido, de acordo com a classificação climática de Köppen (Moreno, 1961). A temperatura média do mês mais quente do ano é de $24,7^{\circ} \mathrm{C}$ e do mês mais frio, de $13,8^{\circ} \mathrm{C}$, sendo a precipitação média anual normal de $1712,4 \mathrm{~mm}$ bem distribuída no ano porém pode apresentar anos bastante secos, como o de 1917 (640 mm) a chuvosos, como 1941 (2953 mm) (Heldwein et al., 2009).

A área em que foi conduzido o experimento localiza-se em terreno suavemente ondulado, sem problemas de erosão e havia sido cultivada com milho no verão anterior. Para a instalação do experimento o solo não foi revolvido procedendose à dessecação da vegetação invasora com herbicida glifosato na dose de $2 \mathrm{~L} \mathrm{ha}^{-1}$ com antecedência de cerca de 40 dias e a limpeza da superfície do solo no dia anterior, através de leve raspagem superficial.

$\mathrm{O}$ solo da área experimental pertence à unidade de mapeamento São Pedro, sendo classificado como Argissolo Vermelho Distrófico arênico (PVd) (Streck et al., 2002).

Para as determinações da curva característica e da função da condutividade hidráulica do solo em campo, o método preconiza a aplicação de diferentes vazões em pontos distintos na superfície do solo. Assim, em pontos isolados foram aplicadas, através de gotejadores, as vazões de 2, 4 e $8 \mathrm{~L} \mathrm{~h}^{-1}$. Para agilizar a obtenção das variáveis necessárias foi montado um sistema multigotejador, composto de três tubos flexíveis, espaçados 3,0 $\mathrm{m}$ entre si, nos quais foram fixados três gotejadores de mesma vazão e na mesma linha, espaçados 2,0 m entre si; desta forma, 
cada uma das duas determinações experimentais realizadas era composta por nove parcelas de $6 \mathrm{~m}^{2}$, totalizando seis repetições para cada vazão.

O sistema multigotejador foi construído para facilitar as determinações simultâneas dos parâmetros do solo proposto pelo "método do gotejador" (principalmente para o parâmetro sorptividade) em que se deseja estimar a condutividade hidráulica e a curva de retenção de água no solo (Shani et al., 1987). A linha principal foi composta de tubo flexível com $10 \mathrm{~m}$ de comprimento e $2,54 \mathrm{~cm}$ (1") de diâmetro e a secundária com $8 \mathrm{~m}$ de comprimento de e 1,27 cm de diâmetro (1/2"). Portanto, para cada linha com três gotejadores de vazões definidas teve-se $6 \mathrm{~m}$ com $1 \mathrm{~m}$ de bordadura em cada extremidade; na primeira linha as vazões eram de $2 \mathrm{~L} \mathrm{~h}^{-1}$, na segunda $4 \mathrm{~L} \mathrm{~h}^{-1}$ e na terceira a vazão foi de $8 \mathrm{~L} \mathrm{~h}^{-1}$ (Figura 1 ).

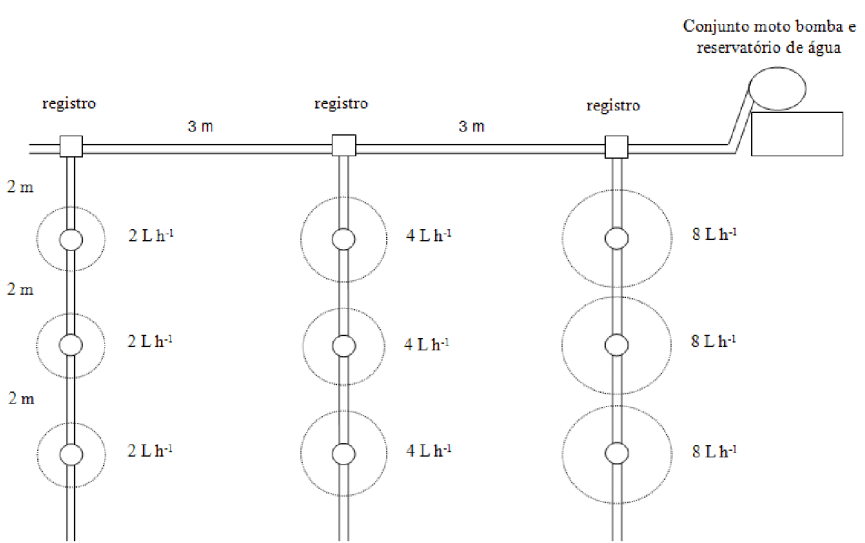

Figura 1. Croqui e disposição do sistema multigotejador

Depois de instalado o sistema multigotejador no campo e com auxílio de um cronômetro, iniciou-se a irrigação e se mediu, em intervalos de $5 \mathrm{~min}$, a distância do ponto de fornecimento d'água até a borda da área saturada para todas as vazões, simultaneamente, até ocorrer o equilíbrio dinâmico. Na Figura 2A detalha-se o avanço da frente de molhamento " $x$ " em função do tempo $\mathrm{t}$, em intervalos de $5 \mathrm{~min}$, até ocorrer $\mathrm{o}$ equilíbrio dinâmico o qual é caracterizado quando os aumentos dos valores do raio da frente de molhamento nos intervalos de $5 \mathrm{~min}$ se tornam quase nulos. Onde ocorreu o equilíbrio dinâmico a irrigação foi interrompida na respectiva linha de gotejadores e imediatamente se mediu a área saturada com o auxílio das réguas com indicação em cm e resolução em mm. Feito isto, passou-se imediatamente à medição das distâncias para o cálculo da sorptividade (S). Para isto foram feitas dez medições em intervalos de $3 \mathrm{~min}$ tomando-se como tempo zero o momento do equilíbrio dinâmico na linha (Figura 2B). Procedeu-se a esta medição em todas as repetições das três diferentes vazões, com ajuda das duas réguas perpendiculares medindo-se da frente de molhamento nas direções norte e sul e leste e oeste para obter o valor médio do raio (x) (Figura 2B).

Considerando que o sistema possuía três conjuntos de três gotejadores permitindo aplicar vazões constantes (Q) de 2, 4 e $8 \mathrm{~L} \mathrm{~h}^{-1}$ de forma contínua em uma mesma área (A) em formato circular, foi então possível obter pares de fluxo $(q=Q / A)$ nas diferentes vazões e também o respectivo valor inverso de raio médio da área saturada em equilíbrio dinâmico $\left(\mathrm{r}^{-1}=(\mathrm{A} / \pi)^{-0,5}\right)$;
A.
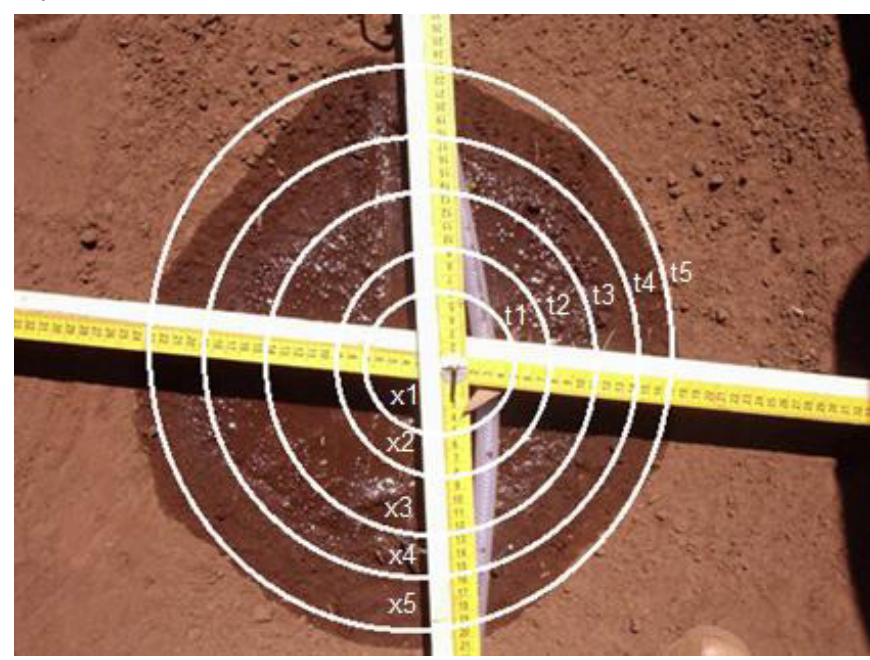

B.
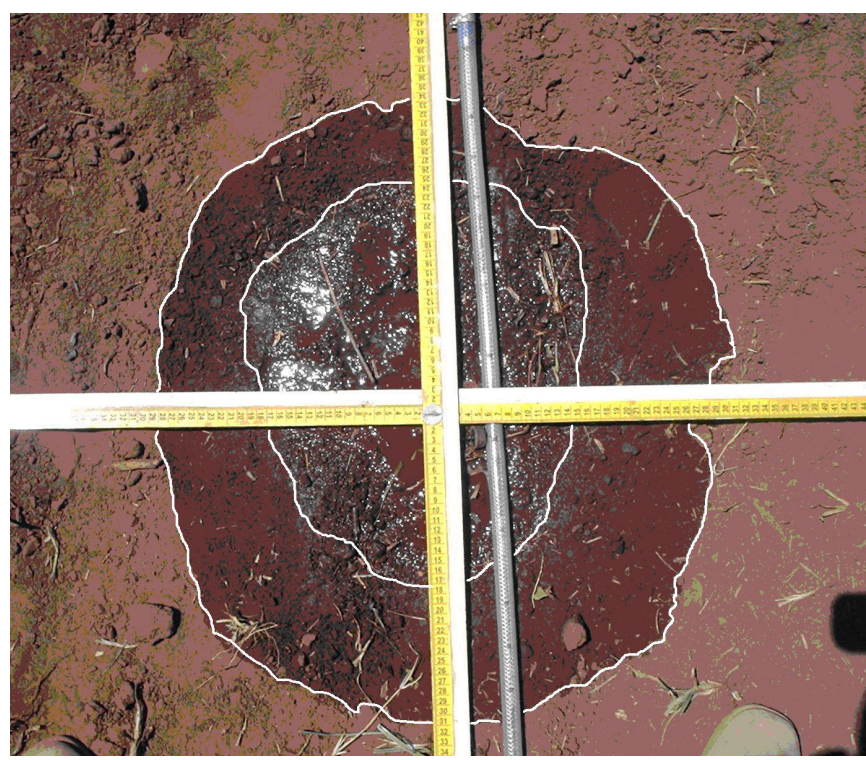

Figura 2. Detalhe do avanço da frente de molhamento $x$ em função do tempo $t(A)$ e amedição da área saturada (B)

portanto, uma equação de regressão linear foi ajustada com esses pares de pontos permitindo estimar os valores de condutividade hidráulica saturada $\left(\mathrm{K}_{\mathrm{s}}\right)$.

O parâmetro do solo $\alpha$ que é representada pela função $\mathrm{K}(\mathrm{h})=$ $\mathrm{e}^{(\mathrm{a}, \mathrm{b})}$ que, relacionada à sorptividade do solo, traduz a capacidade de um solo adsorver água por ação da capilaridade e representa a taxa de redução na condutividade hidráulica com a redução do potencial matricial. Esses valores variam de acordo com a faixa do conteúdo de água no solo ou com o regime de umidade ou de potencial matricial, dependendo da distância em relação ao gotejador. Seus valores podem ser obtidos conhecendo-se a faixa do conteúdo de água no solo ou de potencial a certa distância do gotejador, pela função logarítmica $\ln [\mathrm{K}(\mathrm{h})]$ x h, sendo $\mathrm{K}(\mathrm{h})$ função condutividade hidráulica saturada e h é o potencial matricial de água no solo, que pode ser derivada da curva de retenção (Genuchten, 1980; Dourado Neto et al., 1993). Neste caso, a é a declividade da curva no ponto correspondente ao valor médio de $\mathrm{h}$ e $\mathrm{K}_{\mathrm{s}}$ é o intercepto da tangente com o eixo $\ln [\mathrm{K}(\mathrm{h})]$ de acordo com o método da declividade média móvel de Moldrup et al. (1989). 
Portanto, foi necessário conhecer o conteúdo volumétrico de água do solo seco $\left(\theta_{\mathrm{i}}\right)$ e saturado $\left(\theta_{\mathrm{s}}\right)$ nos primeiros centímetros do solo, o que foi determinado pelo método gravimétrico, com amostras coletadas até a profundidade de $2 \mathrm{~cm}$. Para as vazões aplicadas sobre o solo inicialmente secado ao $\operatorname{ar}\left(\theta_{\mathrm{i}}=\theta_{\mathrm{r}}\right)$ e após se ter verificado o equilíbrio dinâmico, o avanço da frente de molhamento medido em distância(x) foi usado para estimar o valor da sorptividade (S). Como a área é em formato circular, os raios medidos com régua nas quatro direções permitiram obter o valor médio x representativo, para cada intervalo de regressão entre os valores de $\mathrm{x}$ e $\mathrm{t}^{1 / 2}$, que foram tabulados. Com esses pares determinou-se a declividade da reta de regressão $b_{1}$ e, consequentemente, o valor de Sfoi calculado com a relação $\mathrm{S}=\mathrm{b}_{1}(\theta \mathrm{s}-\theta \mathrm{i})^{-1}$.

De posse desses dados e os substituindo na Eq. 1, o valor de $\eta$ foi obtido algebricamente, para o solo considerado.

$$
\eta=0,5\left\{\left(C_{2}+C_{1}\right)+\left[\left(C_{2}+C_{1}\right)^{2}-4 C_{2}\right]^{0,5}\right\}
$$

sendo:

$$
\begin{array}{ll}
\mathrm{C}_{2} & -5 \mathrm{~b}(\theta \mathrm{s}-\theta \mathrm{r})(\mathrm{n}+2) /(3 \mathrm{n}+8) \mathrm{S}^{2} \\
\mathrm{C}_{1} & -(3 \mathrm{n}+10) /(3 \mathrm{n}+8) \\
\mathrm{n} & - \text { número inteiro, permeabilidade de um meio poroso } \\
\mathrm{S} & \text { - sorptividade do solo, } \mathrm{cm} \mathrm{h}^{-0,5}
\end{array}
$$

Os valores de $\beta$ e hw nas Eqs. 2 e 3, foram determinados, respectivamente:

$$
\begin{gathered}
\beta=\frac{(\eta-2)}{(\eta+2)} \quad \eta>2 \\
h w=\frac{(1-\eta)}{\alpha \eta}
\end{gathered}
$$

Deste modo, todas as informações necessárias para determinar a condutividade hidráulica (Eq. 4) e a curva de retenção (Eq. 5) estavam disponíveis.

$$
\begin{gathered}
K(h)=K s\left(\frac{h w}{h}\right)^{\eta} \quad h<h w \\
\theta(h)=(\theta s-\theta r)\left(\frac{h w}{h}\right)^{\beta}+\theta r \quad h<h w
\end{gathered}
$$

sendo:

Ks - condutividade hidráulica saturada, $\mathrm{cm} \mathrm{h}^{-1}$

hw - potencial matricial de entrada de ar, cm de coluna de $\mathrm{H}_{2} \mathrm{O}$

$\theta \quad$ - conteúdo volumétrico de água no solo, $\mathrm{cm}^{3} \mathrm{~cm}^{-3}$

$\theta \mathrm{r} \quad$ - conteúdo residual de água no solo seco $(\mathrm{k}=0), \mathrm{cm}^{3} \mathrm{~cm}^{-3}$

$\theta \mathrm{s} \quad$ - conteúdo de água do solo saturado, $\mathrm{cm}^{3} \mathrm{~cm}^{-3}$

$\eta \quad$ - parâmetro de solo

Seki (2007) desenvolveu um programa computacional de código aberto que usa procedimento totalmente automático para estimar os parâmetros de solo e a curva característica de retenção de água no solo selecionando, automaticamente, as estimativas iniciais baseadas em observações de campo. Os três modelos de Brooks \& Corey (1964), Genuchten (1980) e Kosugi (1996) apresentados na Tabela 1, foram ajustados aos pontos da curva de retenção usando-se a metodologia de Seki (2007) para obter os parâmetros da curva característica de retenção de água no solo em todos os locais de amostragem, utilizando-se os dados determinados em laboratório, considerada a metodologia padrão, quanto os dados obtidos em campo (experimento).

Tabela 1. Modelos para ajuste das curvas de retenção de água no solo

\begin{tabular}{lc}
\hline \multicolumn{1}{c}{ Modelos } & \multicolumn{1}{c}{ Equações } \\
Brooks \& Corey (1964) & $\mathrm{S}_{\mathrm{e}}=\left(\frac{\mathrm{h}}{\mathrm{h}_{\mathrm{b}}}\right)^{-\lambda} \quad \mathrm{h}>\mathrm{h}_{\mathrm{b}}$ \\
& $\mathrm{S}_{\mathrm{e}}=1 \quad \mathrm{~h} \leq \mathrm{h}_{\mathrm{b}}$ \\
\hline Genuchten (1980) & $\mathrm{S}_{\mathrm{e}}=\left[\frac{1}{1+(\alpha \mathrm{h})^{\mathrm{n}}}\right]^{\mathrm{m}} \mathrm{m}=1-\frac{1}{\mathrm{n}}$ \\
\hline Kosugi (1996) & $\mathrm{S}_{\mathrm{e}}=\mathrm{Q}\left[\frac{\ln \left[\left(\mathrm{h} / \mathrm{h}_{\mathrm{m}}\right)\right.}{\sigma}\right]$ \\
\hline
\end{tabular}

$\mathrm{h}$ - tensão de água no solo, cm de água; $\mathrm{h}_{\mathrm{b}}$ - potencial matricial de entrada de ar $\left(\mathrm{h}_{\mathrm{w}}\right)$ $\mathrm{cm}$ de água; $\theta$ - conteúdo volumétrico de água, $\mathrm{cm}^{3} \mathrm{~cm}^{-3}$; Se - conteúdo efetivo de água; $\mathrm{Q}(\mathrm{x})$ - função da distribuição normal cumulativa complementar; $\theta \mathrm{s}$ - conteúdo volumétrico de água no solo saturado, $\mathrm{cm}^{3} \mathrm{~cm}^{-3} ; \theta r$ - conteúdo volumétrico de água residual, $\mathrm{cm}^{3} \mathrm{~cm}^{-3}$ k - condutividade hidráulica, $\mathrm{cm} \mathrm{min}^{-1} ; \theta(\mathrm{h})$ - retenção de água do solo em função do potencial matricial, $\mathrm{cm}^{3} \mathrm{~cm}^{-3} ; \mathrm{m}, \mathrm{n}, \alpha\left(\mathrm{cm}^{-1}\right), \lambda$ e $\sigma$ - parâmetro do solo

Considerando que as curvas características obtidas pelas duas metodologias de determinação (padrão de laboratório e experimento de campo) não são semelhantes (Oliveira, 1988) é necessário realizar sua correção, procedimento este realizado através de funções exponenciais que relacionam os pares de valores de umidade volumétrica obtidos com as duas metodologias para um mesmo potencial matricial, utilizando-se o software computacional "table curve 2D" (Systat Software Inc).

\section{Resultados E Discussão}

O conteúdo volumétrico de água do solo determinado antes do início do experimento ( $\theta \mathrm{i}$ ), foi igual a $\theta \mathrm{i}=\theta \mathrm{r}=0,09$ $\left(\mathrm{cm}^{3} \mathrm{~cm}^{-3}\right)$. O conteúdo de água do solo saturado obtido a partir das amostras coletadas logo após o experimento, foi de $\theta \mathrm{s}=0,43\left(\mathrm{~cm}^{3} \mathrm{~cm}^{-3}\right)$.

Estudo proposto por Oliveira (1988) indica que para uma vazão de 7,343 $\mathrm{L} \mathrm{h}^{-1}$ a área saturada chegou a $962,11 \mathrm{~cm}^{2} \mathrm{e}$ o tempo de irrigação gasto até o equilíbrio foi de $135 \mathrm{~min}$, aproximadamente. Para este trabalho o mesmo volume alcançou uma área de $1134,11 \mathrm{~cm}^{2}$ para o tempo de $210 \mathrm{~min}$ até o equilíbrio dinâmico da frente de molhamento mostrando que há diferenças de resposta de solos com classes texturais diferentes para o equilíbrio dinâmico. Na literatura também aparecem diferenças quando se considera o equilíbrio dinâmico em profundidade. Barros et al. (2009) utilizaram o solo classificado como Nitossolo Vermelho, segundo a classificação brasileira de solos (EMBRAPA, 1997) e a estabilização do crescimento do bulbo formado no perfil do solo ocorreu após $180 \mathrm{~min}$ nos sistemas superficiais, contrariando os dados de Nogueira et al. (2000) que, trabalhando em solo franco argiloso, confirmaram 
estabilização após $24 \mathrm{~h}$. Essas diferenças de tempo para a estabilização do crescimento da frente horizontal superficial de molhamento entre as medidas no trabalho atual e as dos demais autores são devidas, sem dúvida, às diferentes textura e estrutura de cada classe de solo.

$\mathrm{Na}$ Figura 3 constata-se a área $\left(\mathrm{cm}^{2}\right)$ da frente de molhamento em função do tempo (min) desde o início da irrigação até o início do equilíbrio dinâmico para as vazões de 2,4 e $8 \mathrm{~L} \mathrm{~h}^{-1}$ nas repetições 1,2 e 3 . Verifica-se que os resultados são semelhantes entre as repetições para as vazões de 4 e $8 \mathrm{~L}$ $\mathrm{h}^{-1}$, enquanto para a vazão de $2 \mathrm{~L} \mathrm{~h}^{-1}$ na repetição da área de molhamento no equilíbrio dinâmico foi um pouco maior, o que pode ser decorrente da variabilidade espacial das propriedades físico-hídricas do solo (Montenegro \& Montenegro, 2006).

A.

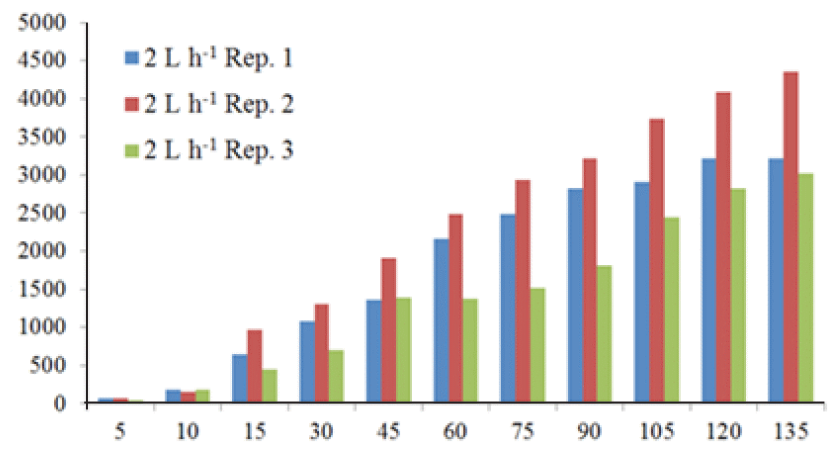

B.

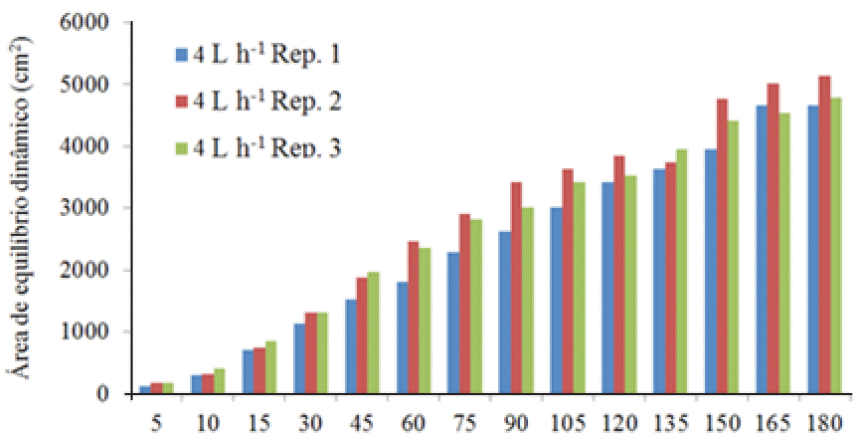

C.

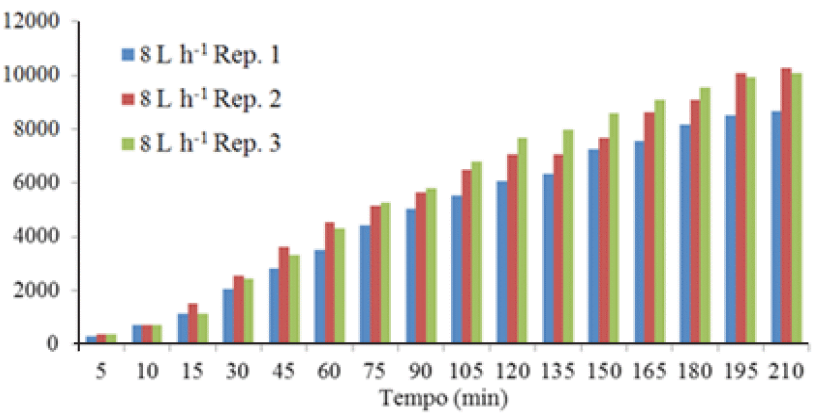

Figura 3. Tempo do avanço da frente de molhamento desde o início da irrigação até o equilíbrio dinâmico para as vazões de 2 , 4 e $8 \mathrm{~L} \mathrm{~h}^{-1}$ dos gotejadores, nas repetições 1,2 e 3

A condutividade hidráulica saturada $(\mathrm{Ks})$ obtida em campo foi igual a $5,59 \mathrm{~cm} \mathrm{~h}^{-1}$. Este resultado foi obtido com as três vazões aplicadas de 2,4 e $8 \mathrm{~L} \mathrm{~h}^{-1}$ e suas respectivas áreas em equilíbrio dinâmico e do ajuste de uma reta de regressão, sendo a variável independente o inverso do raio médio medido da área saturada. $\mathrm{O}$ valor da declividade da reta de regressão, igual a $34,81 \mathrm{~cm}^{2} \mathrm{~h}^{-1}$, é o aumento médio da área de molhamento com o tempo.

Montenegro \& Montenegro (2006) afirmaram que a condutividade hidráulica saturada $(\mathrm{Ks})$ da camada subsuperficial de solo de textura franca e franco-arenosa, variou de 0,07 a $93 \mathrm{~m} \mathrm{~d}^{-1}$, com média igual a $7,13 \mathrm{~m} \mathrm{~d}^{-1}$ para a camada de solo $0-15 \mathrm{~cm}$. A condutividade hidráulica obtida neste trabalho $\left(1,34 \mathrm{~m} \mathrm{~d}^{-1}\right)$ também em solo franco arenoso pode ser aceita como correta mesmo porque a variação entre as repetições foi relativamente pequena. Zuquette \& Palma (2006) concluíram, em seus estudos, que a condutividade hidráulica saturada $(\mathrm{Ks})$ variou de 0,0013 a $0,031 \mathrm{~m} \mathrm{~d}^{-1}$, para os materiais inconsolidados residuais de basalto, entre 0,17 a $2,72 \mathrm{~m} \mathrm{~d}^{-1}$, para os materiais inconsolidados residuais arenosos e $0,39 \mathrm{~m} \mathrm{~d}^{-1}$, para os materiais inconsolidado saluvionares. Conforme Pinheiro \& Teixeira (2009) utilizando o modelo BEST, que é baseado no método semifísico de Beerkan para determinação dos parâmetros das curvas da condutividade hidráulica $K(\theta)$ e de retenção $h(\theta)$ o maior valor encontrado da condutividade hidráulica saturada foi obtido na mata nativa $22,91 \mathrm{~m} \mathrm{~d}^{-1} \mathrm{e}$ o menor, na área de milho com plantio convencional $1,123 \mathrm{~m} \mathrm{~d}^{-1}$ em solo franco siltoso.

Considerando a relação dos valores de raiz quadrada do tempo $\left(\mathrm{t}^{0,5}\right)$ e do avanço da frente de molhamento $\mathrm{x}$, a análise de regressão resultou em diferentes valores da declividade da curva (b) para as três vazões do experimento. Para a vazão de $2 \mathrm{~L} \mathrm{~h}^{-1}$, a declividade da curva $\left(\mathrm{b}_{2}\right)$ foi de $0,9417 \mathrm{~cm}^{2} \mathrm{~h}^{-1}$, para $\mathrm{b}_{4}$, correspondente à vazão de $4 \mathrm{~L} \mathrm{~h}^{-1}$, a declividade da reta foi de $1,014 \mathrm{~cm}^{2} \mathrm{~h}^{-1}$ e para a vazão de $8 \mathrm{~L} \mathrm{~h}^{-1}$, a declividade da curva $\left(b_{8}\right)$ foi de $1,074 \mathrm{~cm}^{2} \mathrm{~h}^{-1}$, confirmando que ocorre aumento do avanço horizontal do molhamento com o aumento da vazão (Montenegro \& Montenegro, 2006; Souza \& Matsura, 2004).

A Figura 4 representa o detalhe da transição entre a área saturada e a frente de molhamento. Quando o equilíbrio

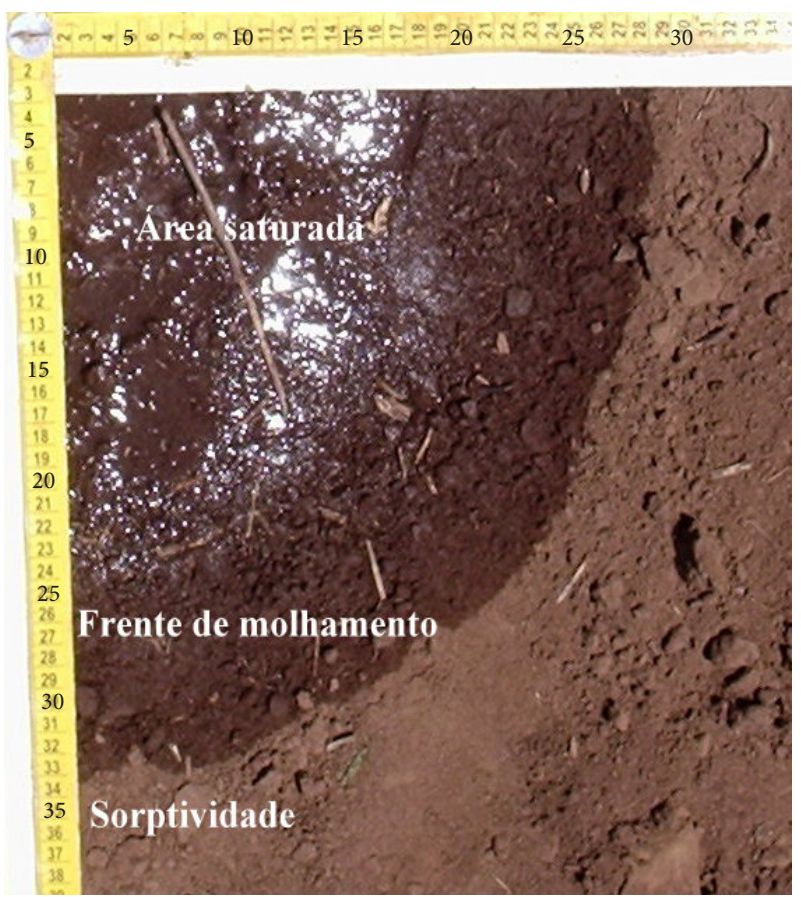

Figura 4. Detalhe da transição entre a área saturada e a frente de molhamento 
dinâmico se estabelece, é importante conhecer o parâmetro do solo denominado sorptividade (S). O sistema multigotejador foi útil para este cálculo devido às repetições simultâneas que o equipamento oferece perfazendo a média das três medições para a sorptividade igual a $2,68 \mathrm{~cm} \mathrm{~h}^{-0,5}$, nas vazões de $2,4 \mathrm{e}$ $8 \mathrm{~L} \mathrm{~h}^{-1}$, diminuindo, assim, os erros de leitura e proporcionando um valor médio representativo para se estimar os parâmetros físicos e hídricos propostos no método.

$\mathrm{O}$ valor calculado da sorptividade foi de $2,48 \mathrm{~cm} \mathrm{~h}^{-0,5}$, para a vazão de $2 \mathrm{~L} \mathrm{~h}^{-1}$, representada aqui por $\mathrm{S}_{2}$. Para a vazão dos gotejadores de $4 \mathrm{~L} \mathrm{~h}^{-1}$ a sorptividade $\left(\mathrm{S}_{4}\right)$ foi igual a $2,74 \mathrm{~cm} \mathrm{~h}^{-0,5}$ e para a vazão de $8 \mathrm{~L} \mathrm{~h}^{-1} \mathrm{o}$ valor da sorptividade $\left(\mathrm{S}_{8}\right)$ foi igual a $2,82 \mathrm{~cm} \mathrm{~h}^{-0,5}$, perfazendo uma média das três medições de vazão igual a 2,68 $\mathrm{cm} \mathrm{h}^{-0,5}$. Em estudo conduzido por Souza et al. (2008) foi estimada a sorptividade em três tipos de Latossolo Amarelo e três tipos de Neossolo Flúvico tendo-se constatado que a sorptividade variou entre 284 a 745,2 e 176,4 a 892,8 cm $\mathrm{h}^{-0,5}$, nos solos desses dois grupos, respectivamente. Os autores salientam que os valores de S e Ks aumentam com o teor de areia identificando, como mais permeável, o Latossolo Amarelo. As medidas "in situ" da sorptividade foram influenciadas pelas condições de contorno definidas para a área experimental, como ausência de declividade, de vegetação e torrões na superfície do solo, obtendo-se um tamanho uniforme da frente de molhamento; ressalta-se que a área estava com solo destorroado e nivelado.

Na validação do experimento em campo foram consideradas as curvas características de retenção de água para o solo da área experimental (Argissolo Vermelho Distrófico arênico - PVd), determinadas em laboratório, para as camadas de 0 a $15 \mathrm{~cm}$ (Figura 5A) e de 15 a $30 \mathrm{~cm}$ de profundidade (Figura 5B). Usaram-se amostras de solo com estrutura não deformada em aneis de aço, submetidas à mesa de tensão, ao aparelho de placas porosas de Richards e ao aparelho de determinação do potencial de água, WP4, conforme Richards (1965), sendo utilizadas as equações de ajuste de Brooks \& Corey (1964), Genuchten (1980) e Kosugi (1996). A equação de Kosugi (1996) foi a que melhor se ajustou aos dados de 0 a $15 \mathrm{~cm}$ de profundidade com valor de $\mathrm{R}^{2}$ igual a 0,972 . Para a profundidade de 15 a $30 \mathrm{~cm}$ as equações de Genuchten (1980) e Kosugi (1996) apresentaram ajuste melhor, com valores de $\mathrm{R}^{2}$ iguais a 0,978 e 0,984 , respectivamente.

Para o valor médio de sorptividade determinado em campo de 2,68 $\mathrm{cm} \mathrm{h}^{-0,5}$, o valor $\eta=2,22$ foi obtido pela aplicação da Eq. 1. Também os valores de $\beta=0,44 \mathrm{e} \mathrm{hw}=-2,29 \mathrm{~cm}$ de $\mathrm{H}_{2} \mathrm{O}$ resultaram dos calculados pelas Eqs. 2 e 3, respectivamente. Usando esses valores estimados dos parâmetros do solo, a curva característica de retenção de água no solo pôde ser determinada em campo, sendo representada na Figura 6 e na Eq. 5 com os seguintes coeficientes como resultado:

$$
\theta(\mathrm{h})=(0,43-0,09)\left(-2,29 \mathrm{~h}^{-1}\right)^{0,44}+0,09
$$

ou, simplificando

$$
\theta(h)=0,489|\mathrm{~h}|^{-0,44}+0,09 \quad \mathrm{~h}<-2,29
$$

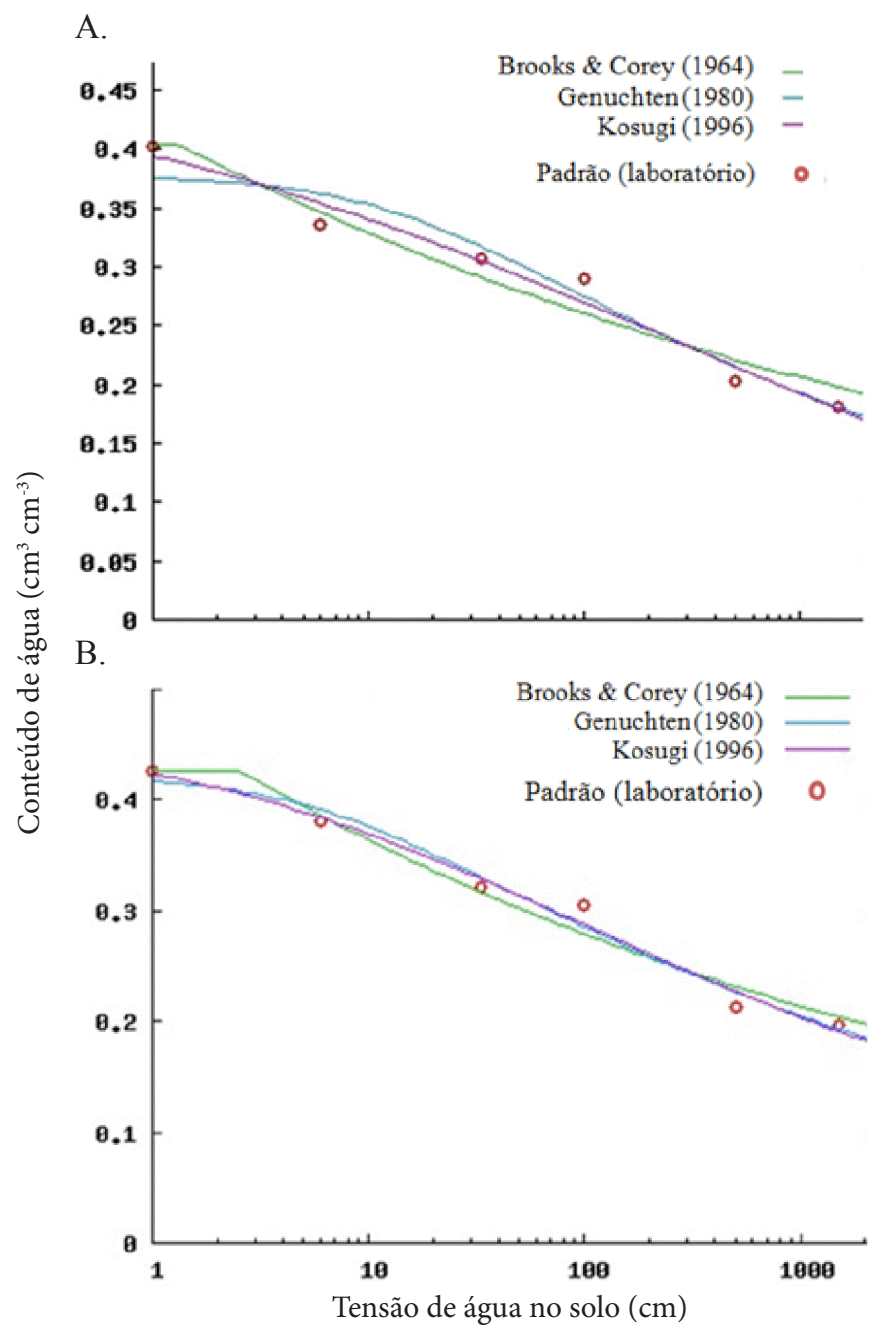

Figura 5. Curva característica de retenção de água no solo Argissolo (PVd), padrão, na camada de 0-15 (A) e de $15-30 \mathrm{~cm}(\mathrm{~B})$ de profundidade determinada em laboratório e ajustada às equações de Brooks \& Corey (1964), Genuchten (1980) e Kosugi (1996)

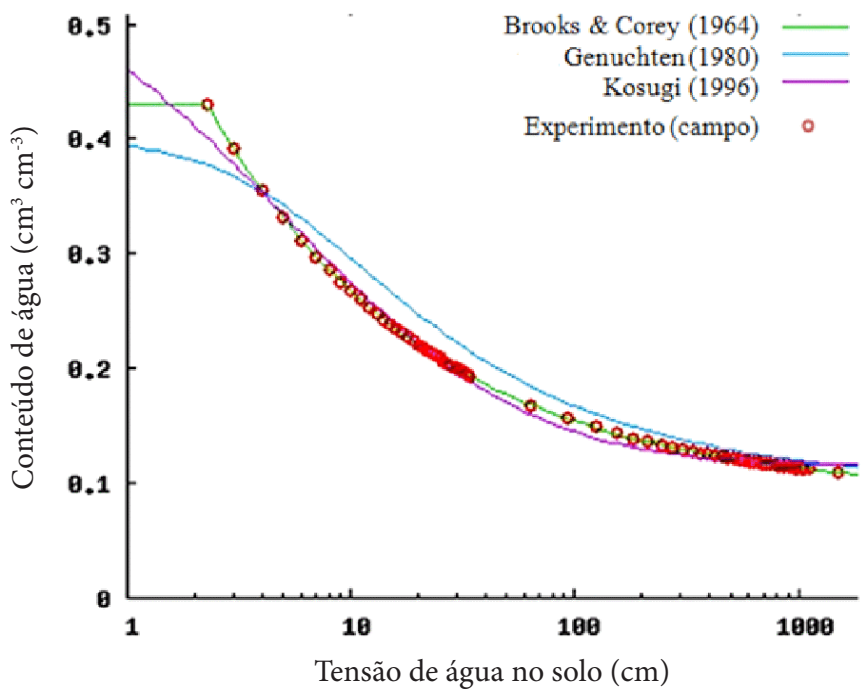

Figura 6. Curva característica de retenção de água no solo Argissolo (PVd) obtida a partir dos dados de campo, ajustada às equações de Brooks \& Corey (1964), Genuchten (1980) e Kosugi (1996) 
sendo:

$\theta(h)$ - conteúdo de água no solo em função da tensão da água no solo, $\mathrm{cm}^{3} \mathrm{~cm}^{-3}$

h $\mathrm{h}$ - módulo da tensão de água no solo, $\mathrm{cm} \mathrm{H}_{2} \mathrm{O}$

Na Figura 6 percebe-se que a primeira entrada de ar referente à drenagem dos macroporos está aproximadamente a $3 \mathrm{~cm}$ de tensão de $\mathrm{H}_{2} \mathrm{O}$ e é relacionada à presença da fração do solo composta por areia, correspondente a $39 \%$ da massa do solo. Observa-se ainda que o final da drenagem ocorre aproximadamente na tensão de $1000 \mathrm{~cm}$ de $\mathrm{H}_{2} \mathrm{O}$. De acordo com Fredlund \& Xing (1994) o valor de entrada de ar representa o nível de sucção a partir da qual o ar começa a entrar nos maiores poros do solo.

A função da condutividade hidráulica de mesma forma pode ser representada matematicamente com a Eq. 4 com os seguintes coeficientes, resultantes dos valores experimentais obtidos no campo:

$$
\mathrm{K}(\mathrm{h})=5,59\left(-2,29 \mathrm{~h}^{-1}\right)^{2,22}
$$

ou, simplificando

$$
\mathrm{K}(\mathrm{h})=34,82|\mathrm{~h}|^{-2,22} \quad \mathrm{~h}<-2,29
$$

sendo:

$\mathrm{K}(\mathrm{h})$ - condutividade hidráulica, $\mathrm{cm} \mathrm{min}{ }^{-1}$

h - tensão de água no solo, $\mathrm{cm} \mathrm{H}_{2} \mathrm{O}$

A condutividade hidráulica em função do conteúdo de água do solo (Figura 7) resultante para este solo, pode ser descrita pela Eq.10:

$$
K(\theta)=5,59\left[\frac{(\theta-0,09)}{0,34}\right]^{5,04}
$$

sendo:

$\mathrm{K}(\theta)$ - condutividade hidráulica em função do conteúdo volumétrico de água do solo, $\mathrm{cm} \mathrm{min}^{-1}$

$\theta \quad$ - conteúdo volumétrico de água do solo, $\mathrm{cm}^{3} \mathrm{~cm}^{-3}$

O diâmetro da área superficial de molhamento e o tempo determinado para que as áreas saturadas permanecessem em equilíbrio dinâmico foram diferentes da metodologia proposta por Oliveira (1988) em virtude, provavelmente, da variabilidade espacial da textura e da estrutura dos solos (Montenegro \& Montenegro, 2006). A curva de retenção de água no solo obtida em campo (Figura 8) nas baixas tensões aproximou-se dos dados de laboratório. Conforme o conteúdo volumétrico de água no solo decresce com o aumento na tensão, os valores obtidos pelo método em campo e do laboratório se afastaram (Figura 8) sendo que o conteúdo volumétrico de água no solo determinado em campo é menor que o do laboratório. Como no método de campo a determinação da sorptividade após a ocorrência do equilíbrio dinâmico foi realizada em nível da superfície do solo pode ter havido, neste caso, um acréscimo do fluxo de água no solo.

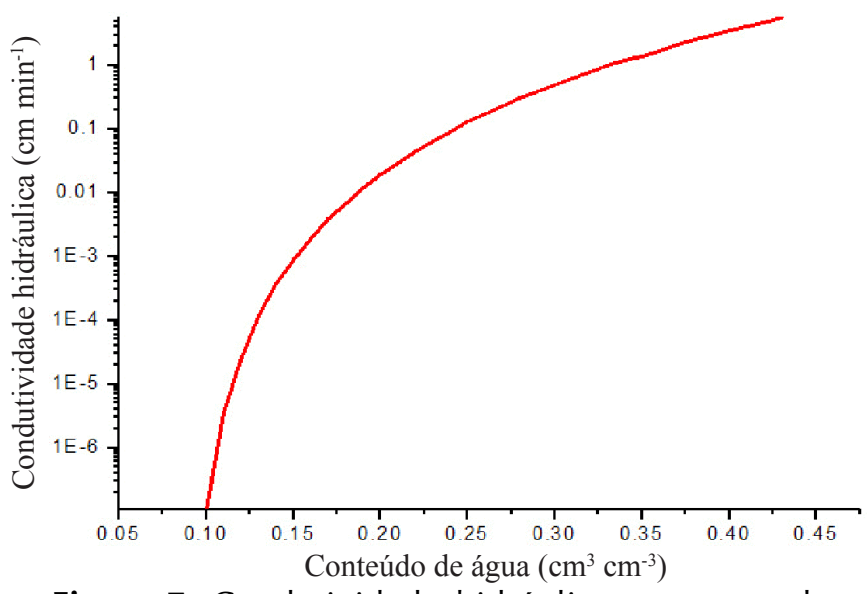

Figura 7. Condutividade hidráulica não saturada do solo Argissolo (PVd) em função do conteúdo volumétrico de água com os dados obtidos no campo

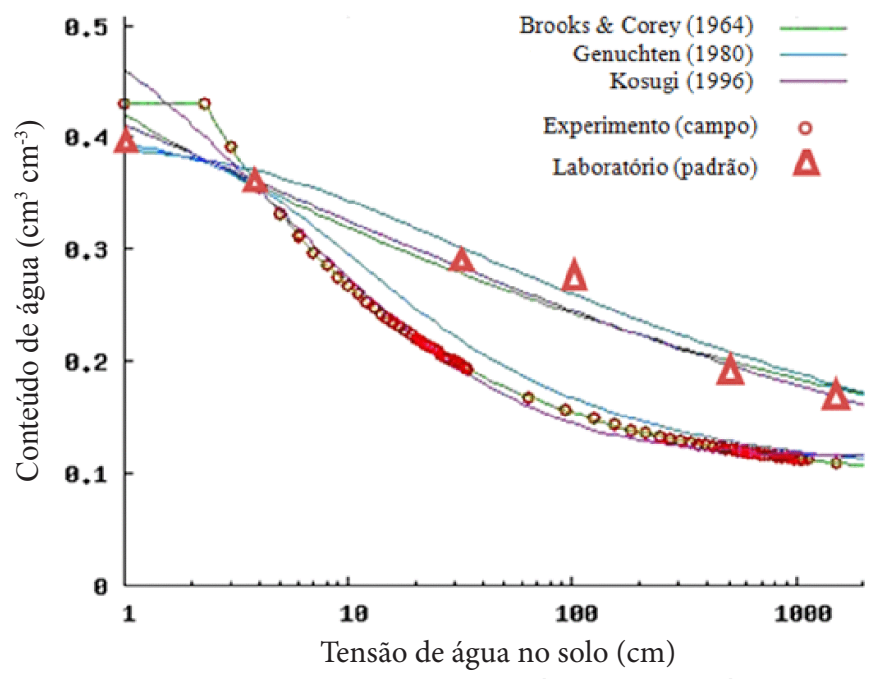

Figura 8. Curvas características de retenção de água no solo Argissolo (PVd), em laboratório (padrão) e experimento (campo), ajustadas às equações de Genuchten (1980), Brooks \& Corey (1964) e Kosugi (1996)

Outro fato que pode ter contribuído para a obtenção de curvas de retenção diferentes em função dos dois métodos de determinação é que a amostra do método de campo foi mantida em estrutura "in situ", contrariamente à do método de laboratório, que foi utilizada amostra não deformada. Isto é relevante visto que as condições do solo no momento da medição refletem a condição atual da área, como estrutura do solo, porosidade, densidade, cobertura vegetal, podendo tais fatores influenciar no fluxo dinâmico de água determinado em campo (Souza et al., 2006; Silva et al., 2012; Lima et al., 2008). Este aumento do fluxo determinado em campo pode ter sido o fator que contribuiu com o decréscimo do conteúdo de água e na inferência de que a curva construída por este método subestima o volume de água no solo.

Classicamente, as curvas de retenção de água no solo são construídas com dados obtidos pela metodologia proposta por Richards (1965) em que são determinados valores de conteúdo de água no solo e respectivo potencial matricial; 
por outro lato, o sistema consta de aparelho de placas porosas de Richards e equipamentos de produção, além de controle de pressão, podendo-se usar tanto amostras não deformadas como deformadas, sendo a determinação em laboratório meio trabalhosa e demanda tempo. Esta é a metodologia padrão adotada e aceita porém sua representatividade depende de uma amostragem cuidadosa.

A metodologia proposta neste estudo mostra a utilidade e a rapidez na elaboração das curvas de retenção e condutividade hidráulica, não demandando muito tempo na sua obtenção, o que é um aspecto importante para quem está no campo e precisa de dados com vista à tomada de decisão. Comparando as curvas elaboradas com os dados obtidos em laboratório e no campo, pela metodologia proposta, sendo as duas ajustadas com as equações de Genuchten (1980), Brooks \& Corey (1964) e Kosugi (1996) verifica-se que são diferentes, o que acarreta restrição ao seu uso direto; no entanto, a relação entre os valores de umidade volumétrica obtidos pelas duas metodologias para um mesmo potencial matricial apresentaram uma relação exponencial entre si em função dos valores de umidade volumétrica obtidos no campo. Esta função exponencial (Figura 9) apresentou alto ajuste aos dados, na faixa de tensões correspondentes a $1 \mathrm{~cm}$ de $\mathrm{H}_{2} \mathrm{O}$ até $1500 \mathrm{~cm}$ de $\mathrm{H}_{2} \mathrm{O}$, o que permitiu corrigir os dados

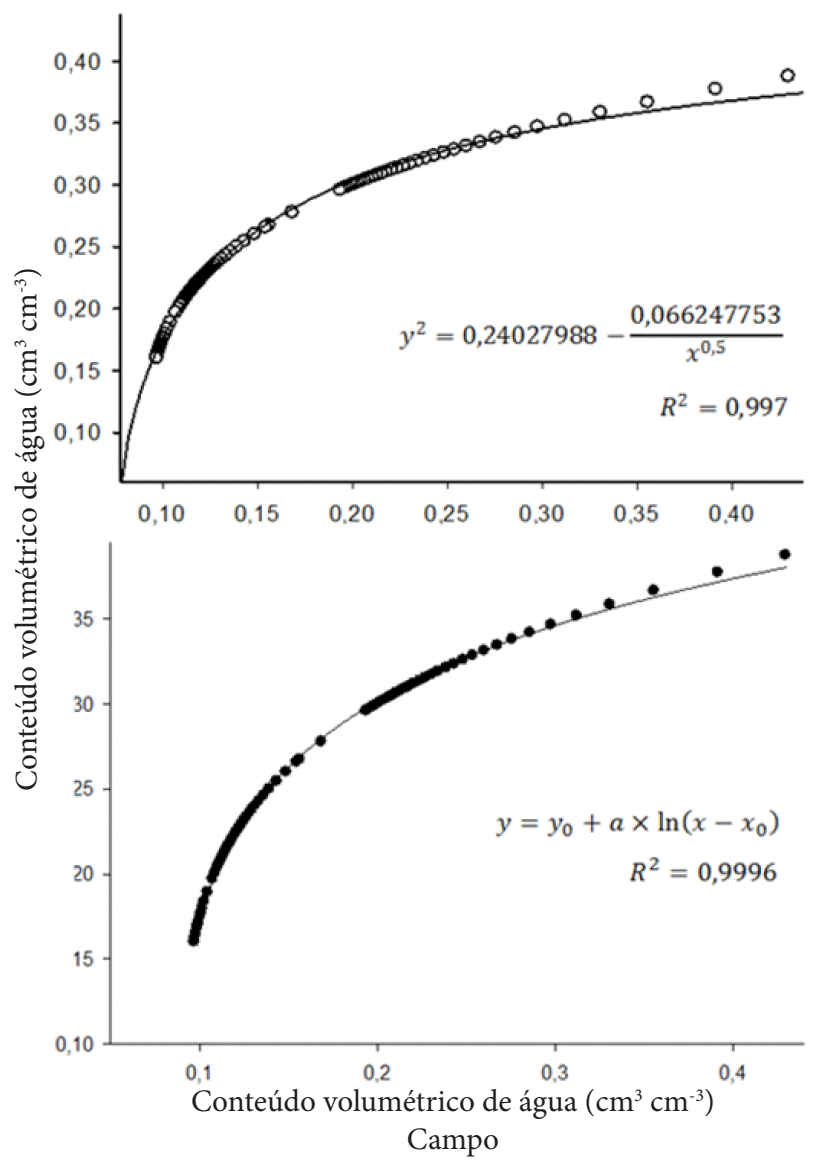

Figura 9. Funções de ajuste para correção de valores de umidade volumétrica obtidos pela metodologia de campo (método alternativo baseado nas variáveis físico-hídricas relacionadas ao avanço da frente de molhamento horizontal na superfície do solo) para um mesmo potencial matricial em laboratório obtidos no campo para seus equivalentes determinados em laboratório pela metodologia padrão.

Portanto, as duas funções ajustadas (Figura 9) podem ser utilizadas para corrigir os valores de umidade volumétrica obtidas em campo pela metodologia, conforme também inferiram Shani et al. (1987). Para Bernardo (2006) existem diferenças consideráveis quando se considera a água disponível no solo determinada pelos métodos de laboratório e de campo. Para as condições em que foi desenvolvido o presente trabalho, em solo PVD, os coeficientes de ajuste a serem utilizados estão representados na Tabela 2.

Tabela 2. Equações e coeficientes de ajuste para correção de valores de umidade volumétrica a campo* para um mesmo potencial matricial em laboratório

\begin{tabular}{clc}
\hline Equações & \multicolumn{1}{c}{ Coeficientes } & $\mathbf{R}^{2}$ \\
$y=y_{0}+a \ln \left(x-x_{0}\right)$ & $\begin{array}{l}y_{0}=0,4577 \\
x_{0}=0,0783\end{array}$ & 0,9996 \\
& $a=0,0740$ & \\
\hline$y^{2}=a-\left(b / x^{0,5}\right)$ & $\begin{array}{l}a=0,24027988 \\
b=0,06624775\end{array}$ & 0,9970 \\
\hline
\end{tabular}

* Método alternativo baseado nas variáveis físico-hídricas relacionadas ao avanço da frente de molhamento horizontal na superfície do solo

\section{Conclusões}

1. O Sistema multigotejador permite obter o maior número de amostragens simultâneas no campo, o que facilita a estimativa de sorptividade (S).

2. Através das medidas feitas com o sistema multigotejador, os cálculos de sorptividade $(\mathrm{S})$, parâmetros do solo $(\eta)$ e $(\beta)$ e do potencial matricial de entrada de ar (hw) são suficientes para obtenção das curvas características de água no solo e da condutividade hidráulica não saturada para o solo Argissolo Vermelho Distrófico arênico (PVd).

3. Os resultados obtidos com os métodos de determinação (laboratório e campo) são diferentes, o que traz restrições ao seu uso direto.

4. As funções exponenciais de ajuste podem ser utilizadas para corrigir os valores de umidade volumétrica obtidos no campo e ser possível predizer a curva característica de retenção de água no solo e da condutividade hidráulica do solo.

\section{Literatura Citada}

Andrade, R. S.; Stone, L. F. Uso do índice S na determinação da condutividade hidráulica não-saturada de solos do cerrado brasileiro. Revista Brasileira de Engenharia Agrícola e Ambiental, v.13, p.376-381, 2009.

Barros, A. C.; Folegatti, M. V.; Souza, C. F.; Santoro, B. L. Distribuição de água no solo aplicado por gotejamento enterrado e superficial. Revista Brasileira de Engenharia Agrícola e Ambiental, v.13, p.700-707, 2009.

Bernardo, S. Manual de irrigação. 8.ed.,Viçosa: UFV, 2006. 625p. Borges, E.; Libardi, P. L. Propriedades hidráulicas de um solo não-saturado utilizando-se o permeâmetro a disco. Scientia Agricola, v.57, p.525-529, 2000.

Brooks, R. H.; Corey, A. T. Hydraulic properties of porous media. Hydrological Paper. Fort Collins: Colorado State University, n.3, 1964. 54p. 
Dourado Neto, D.; Moraes, S. O.; Libardi, P. L. Problemas metodológicos na obtenção da curva de retenção da água pelo solo. Ciência Agrícola, v.50, p.383-392, 1993.

EMBRAPA - Empresa Brasileira de Pesquisa Agropecuária. Centro Nacional de Pesquisa de Solos. Manual de métodos de análise de solo. Rio de Janeiro: EMBRAPA. 1997. 212p.

Fredlund, D. G.; Xing, A. Equations for the soil-water characteristic curve. Canadian Geotech Journal, v.21, p.521-532, 1994.

Genuchten, M. T. van. A closed-form equation for predicting the hydraulic conductivity of unsaturated soils. Soil Science Society of America Journal, v.44, p.892-898, 1980.

Heldwein, A. B.; Buriol, A. G.; Streck, N. A. O clima de Santa Maria. Ciência \& Ambiente, v.38, p.43-58, 2009.

Kosugi, K. Lognormal distribution model for unsaturated soil hydraulic properties. Water Resource Research, v.32, p.2697-2703, 1996.

Lima, C. L. R. de; Pillon, C. N.; Suzuki, L. E. A. S.; Cruz, L. E. C. Atributos físicos de um planos solo háplico sob sistemas de manejo comparados aos docampo nativo. Revista Brasileira de Ciência do Solo, v.32, p.1849-1855, 2008.

Mesquita, M. G. B. F.; Moraes, S. O. A dependência entre a condutividade hidráulica saturada e atributos físicos do solo. Ciência Rural, v.34, p.963-969, 2004.

Moldrup, P.; Rolston, D. E.; Hansen, J. A. Rapid and numerically stable simulation of one dimensional, transient water flow in unsaturated layered soils. Soil Science Society of America Journal, v.148, p.219-226, 1989.

Montenegro, A. A. A.; Montenegro, S. M. G. G. Variabilidade espacial de classes de textura, salinidade e condutividade hidráulica de solos em planície aluvial. Revista Brasileira de Engenharia Agrícola e Ambiental, v.10, p.30-37, 2006.

Moreno, J. A. Clima do Rio Grande do Sul. Porto Alegre: Secretaria da Agricultura e Abastecimento, Diretoria de Terras e Colonização, Seç̧ão de Geografia, 1961.

Nogueira, C. C. P.; Coelho, E. F.; Leão, M. C. Características e dimensões do volume de um solo molhado sob gotejamento superficial e subsuperficial. Revista Brasileira de Engenharia Agrícola e Ambiental, v.4, p.315-320, 2000.

Oliveira, C. A. S. Influence of diking and mulch soil surface treatments on infiltration and runoff as affected by irrigation. 1986. 124p. Logan: Utah State University, Thesis Ph.D

Oliveira, C. A. S. Determinação de condutividade hidráulica e da curva de retenção de água no solo com um método simples de campo. Brasília: EMBRAPA CNPH, 1988. 11p. Circular Técnica 6.

Pinheiro, A.; Teixeira, L. P. Estimativa das curvas de condutividade hidráulica e de retenção a partir de características físicas do solo. Revista de Estudos Ambientais, v.11, p.44-50, 2009.

Reichardt, R.; Timm, L. C. Solo planta atmosfera: Conceitos, processos e aplicações. Barueri: Manole, 2004. 478p.
Reis, E. F.; Faria, P. A. S.; Guariz, H. R.; Effgen, E. M.; Passos, R. R. Efeito do manejo na curva de retenção de água no solo, em duas profundidades distintas. In: X Encontro Latino Americano de Iniciação Científica, e VI Encontro Latino Americano de Pós-Graduação Universidade do Vale do Paraíba. Anais...São José dos Campos, São Paulo, SP, 2006. p.2899-2901.

Richards, L. A. Physical conditions of water in soil. In: Black, C. A.; Evans, D. D.; White, J. L.; Ensminge, L. E.; Clark, F. E. Methods of soil analysis - Physical and mineralogical properties, including statistics of measurements and sampling. Madison: ASASSSA, 1965.p.128-152.

Righes, A. A.; Silva, R. F. Escoamento Superficial, Erosão e contaminação do solo. In: Righes, A. A.; Buriol, G. A.; Boer, N. Água e educação: Princípios e estratégias de uso e conservação. Cap. V, p.137-168. Santa Maria: Centro Universitário Franciscano, 2009. 272p.

Saito, H.; Seki, K.; Simunek, J. An alternative deterministic method for the spatial interpolation of water retention parameters. Hydrology and Earth System Sciences, v.13, p.453-465, 2009.

Seki, K. Swrc fit - A nonlinear fitting program with a water retention curve for soils having unimodal and bimodal pore structure. Hydrology and Earth System Sciences, v.4, p.407-437, 2007.

Shani, U.; Hanks, J. R.; Bresler, E.; Oliveira, C. A. S. A simple field method for estimating the hydraulic conductivity and matric potential water content relations of soils. Soil Science Society of America Journal, v.51, p.298-302, 1987.

Silva, J. R. L. da; Montenegro, A. A. A; Santos, T. E. M. dos. Caracterização física e hidráulica de solos em bacias experimentais do semiárido brasileiro,sob manejo conservacionista. Revista Brasileira de Engenharia Agrícola e Ambiental, v.16, p.27-36, 2012.

Souza, C. F.; Matsura, E. E. Distribuição da água no solo para o dimensionamento da irrigação por gotejamento. Revista Brasileira de Engenharia Agrícola e Ambiental, v.8, p.715, 2004.

Souza, E. S.; Antonino, A. C. D; Jamirillo, R. A.; Netto, A. M. Caracterização hidrodinâmica de solos: Aplicação do método Beerkan. Revista Brasileira de Engenharia Agrícola e Ambiental, v.12, p.128-135, 2008.

Souza, Z. M. de; Marques Júnior, J.; Cooper, M.; Pereira, G. T. Micromorfologia do solo e sua relação com atributos físicos e hídricos. Pesquisa Agropecuária Brasileira. v.41, p.487-492, 2006.

Streck, E. V.; Kämpf, N.; Dalmolin, R. S. D.; Nascimento, P. C.; Schneider, P. Solos do Rio Grande do Sul. Porto Alegre: Ed. EMATER/RS; UFRGS, 2002.108p.

Zuquette, L. V.; Palma, J. B. Avaliação da condutividade hidráulica em área de recarga do aquífero Botucatu. Revista Escola de Minas, v.59, p.81-87, 2006. 